\title{
Chondromyxoid Fibroma of the Ethmoid Sinus
}

\author{
James T. Castle $\cdot$ Mikelle L. Kernig
}

Received: 17 May 2011/Accepted: 10 June 2011/Published online: 21 June 2011

(C) Springer Science+Business Media, LLC (outside the USA) 2011

\section{History}

A 43-year old Black female complained of sinus pressure and was found to have a mass of the right ethmoid sinus.

\section{Radiographic Features}

A T1-weighted fat-suppressed (FS) post-clear coronal magnetic resonance imaging (MRI) study (Fig. 1) revealed right mid-level ethmoid air cells that were expanding the sinus cavity. A slightly lower density signal compared to brain was noted, at 35 Hounsfield units, and was found to be isointense with skeletal muscle. Notably, this coronal

Disclaimer The opinions and assertions expressed herein are those of the authors and are not to be construed as official or representing the views of the Department of the Navy, Department of the Air Force or the Department of Defense. I certify that all individuals who qualify as authors have been listed; each has participated in the conception and design of this work, the writing of the document, and the approval of the submission of this version; that the document represents valid work; that if we used information derived from another source, we obtained all necessary approvals to use it and made appropriate acknowledgements in the document; and that each takes public responsibility for it.

J. T. Castle ( $\bowtie)$

Department Of Oral and Maxillofacial Pathology, Naval

Postgraduate Dental School, NMMPT\&E, Bethesda,

MD 20889-5611, USA

e-mail: James.Castle@med.navy.mil

\section{L. Kernig}

Oral and Maxillofacial Pathology, Wright-Patterson AFB,

Dayton, OH 45433, USA

\section{L. Kernig}

Department of Pathology (Histology), SGQC, 4881 Sugar Maple Drive, Dayton, OH 45433, USA image showed that the mass was not descending from the cranial compartment, thereby effectively ruling out an encephalocele. A T1 axial FS MRI (Fig. 2) showed bone remodeling as the tumor had eroded through the lamina papyracea at the medial wall of the orbit. A fluid attenuated inversion recovery MRI (Fig. 3) indicated the absence of bright signals to indicate hemoglobin, necrosis, calcification, or proteinaceous debris. No edema was noted in the medial rectus muscle and no infiltration of fat of the orbit was identified, suggesting a non-aggressive nature to this lesion.

\section{Diagnosis}

The hematoxylin and eosin stained whole mount histologic section of a representative sample of the surgical specimen (Fig. 4) showed numerous irregularly shaped lobules of tissue with varying zones of cellularity. As shown in Fig. 5, there were more cellular areas consisting of bland appearing, but morphologically heterogeneous cells, including stellate and spindled cells with oval nuclei and eosinophilic cytoplasm with indistinct outlines. These cells were contained within a pale blue myxo-chondroid background stroma of varying tinctorial shades and occasionally arranged in a sieve-like pattern in the more myxoid areas. A subtle microlobular pattern characterized by a cellular periphery surrounding a hypocellular central area was noted as an occasional feature. Sparse numbers of granular appearing, polygonal shaped calcifications were identified within the specimen but not as a prominent feature; nor were they interpreted to be an inclusion or entrapment of the surrounding bone structures. Mitotic figures or cells displaying any amount of cellular atypia were not noted. In addition, there were no foci of distinct 


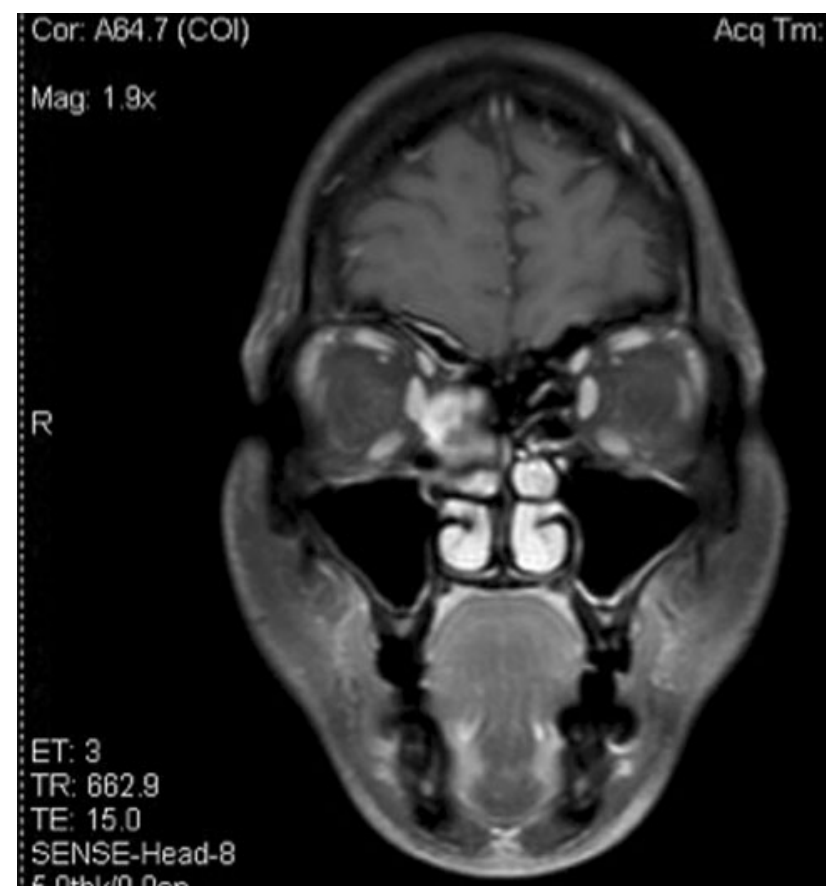

Fig. 1 Coronal T1 weighted MRI demonstrated the right mid-level ethmoid air cells expanding the sinus cavity. The mass does not descend from the cranial cavity, thus ruling out an encephalocele

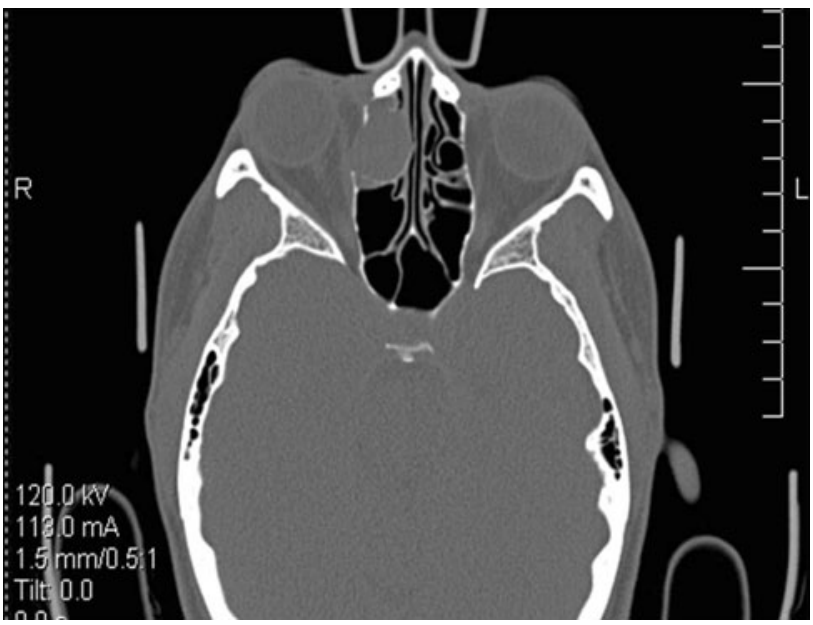

Fig. 2 A T1 axial FS MRI showed bone remodeling as the tumor eroded through the lamina papyracea at the medial wall of the orbit

fragments or islands of hyaline cartilage nor cellular zones of osteoclast-like giant cells along the periphery.

\section{Discussion}

Chondromyxoid fibroma (CMF), believed to be of cartilaginous derivation, [1] is a benign chondroid and myxoid matrix-producing tumor that is occasionally seen

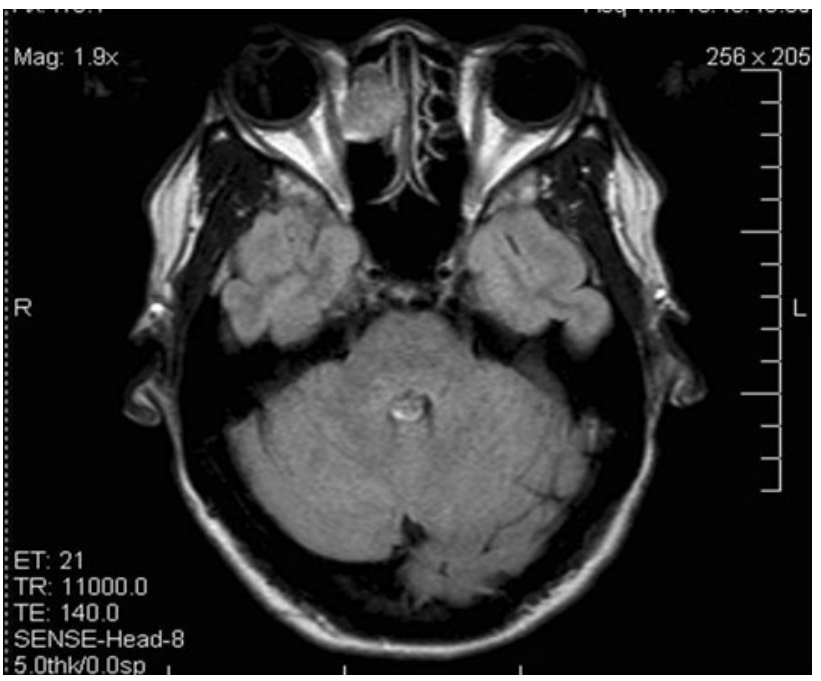

Fig. 3 Fluid-attenuated inversion recovery MRI showed no bright signals to indicate hemoglobin, necrosis, calcification, or proteinaceous debris. No edema was noted in the medial rectus muscle and there was no infiltration of fat of the orbit, thus indicating nonaggressive behavior

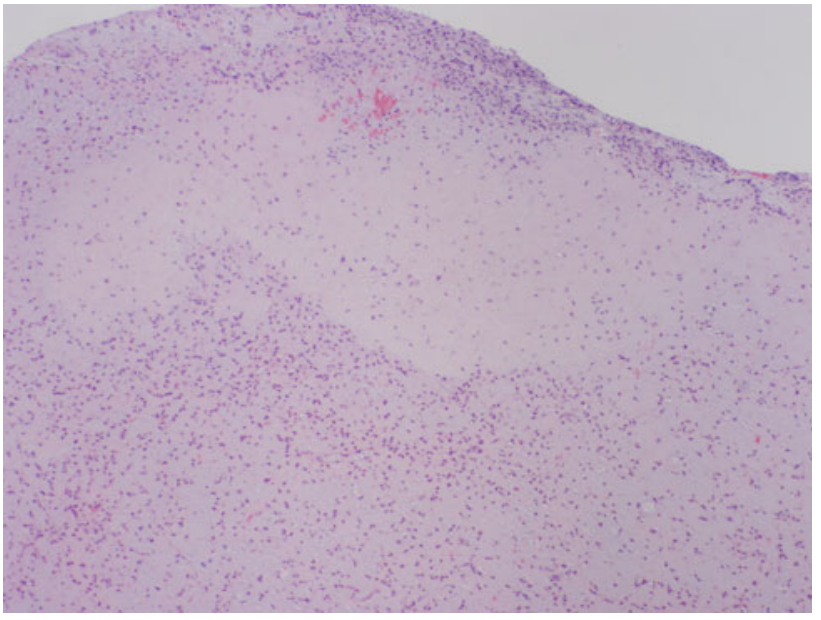

Fig. 4 Low-power examination of the tumor revealed a macrolobular pattern with pale-blue myxoid stroma. Tumor cells are condensed at the periphery of the lobule forming a cellular zone, which is a typical finding of chondromyxoid fibroma

in association with fibrous areas. Accounting for less than $1 \%$ of all bone tumors and $2 \%$ of benign bone tumors [2], CMF presents as a locally destructive lesion usually in the 2nd to 3rd decade, although cases have presented from the 1 st through 8th decades [3]. The male to female ratio is $2: 1$ except when the cranium and facial bones are involved, as women then prevail in a 2:1 ratio [4]. CMF is found most often in the metaphysis around the knee in the proximal tibia, proximal fibula, or distal femur, and rarely in the 


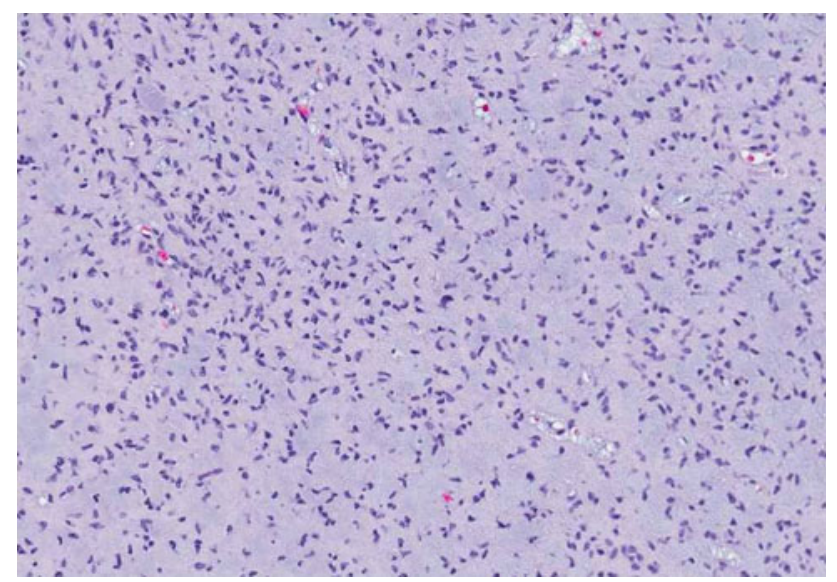

Fig. 5 Closer inspection reveals the characteristic bland appearing stellate and spindle-shaped cells contained in a myxo-chondroid matrix. Mitotic figures were not appreciated

small bones of the hands and feet. Twenty-five percent of cases occur in flat bones to include the skull and facial bones [2]. In most cases, CMF is slow growing and incidentally found on routine radiography for reasons not specifically related to symptoms caused by these lesions. When symptomatic, cases are associated with a long history of chronic local pain (85\%), swelling $(65 \%)$, edema $(65 \%)$, and possibly a palpable soft tissue mass resulting in restriction of movement and, more rarely, pathologic fracture [5].

Typical radiologic findings describe the tumor as an eccentric, loculated, expansile and lytic lesion with welldefined scalloped or lobulated margins, usually with a sclerotic margin of bone. The lobulated contour is sometimes seen with "pseudo-trabeculations" in the tumor mass [6]. Evidence of radiographic calcifications is seen in only $10 \%$ of cases [2] although microscopic calcifications are noted in approximately $25 \%$ of cases [6]. Cortical expansion and focal destruction may be present in cases of CMF but are rare occurrences. The radiographic differential diagnosis includes giant cell tumor, aneurysmal bone cyst, chondroblastoma, and fibrous dysplasia.

CMF may be misdiagnosed as a malignant neoplasm due to its occasional extension into soft tissue and its perceptible recurrence rate. It is for these reasons that CMF should be distinguished from a myxoid variant of chondrosarcoma (which usually displays features of an aggressive neoplasm), since a mischaracterization may have significant patient consequences. Zilmer and Dorfman [3] reported a $22 \%$ initial misdiagnosis rate in $36 \mathrm{CMF}$ cases in their series and pointed out the possible danger of significant erroneous procedures, such as amputation being performed for what is a benign disease. It should also be taken into consideration that cartilaginous tumors of flat bones are more often malignant than benign. Therefore, careful scrutiny of histologic features is critical to resolving the sometimes challenging differential diagnoses which have differing prognoses and require different management. Moreover, it is paramount that all information, including clinical, radiographic, and histologic findings, as well as immunohistochemical results, be taken into consideration.

The classic histologic features of CMF are best defined as macrolobules with spindle-shaped or stellate tumor cells embedded in a myxoid matrix. These spindled or stellate cells are located along the periphery of the lobule imparting an effect of hypercellularity surrounding a hypocellular central area. A microlobular pattern, a combination of both, or no pattern at all, have also been described. Although the nomenclature suggests the presence of chondroid differentiation, it is unusual to find cartilage of the type seen in chondroma or chondrosarcoma and is a focal finding in less than $20 \%$ of CMF cases [5]. Mitotic figures are sparse, accounting for 1-3 cells per 10 high-power fields. The tumor cells, best described as demonstrating pleomorphic features as opposed to cytologic atypia, [5] contain enlarged, hyperchromatic nuclei and are noted to be concentrated in the periphery of the tumor mass. The nucleus to cytoplasmic ratio is not altered, although overall cell size can be enlarged. The lack of atypical mitotic figures in significant numbers may be a useful criterion for separating CMF from chondrosarcoma [5].

The histologic differential diagnosis of CMF includes, most importantly, myxoid chondrosarcoma and chondroblastoma [7]. Chondrosarcomas grow in lobules much like CMF, but are more distinct and occasionally coalesce. These tumors generally contain plump, bizarre cells with multiple nuclei, which is also in contrast with CMF. Myxoid chondrosarcoma shows a bubbly appearance to the stroma along with degenerative and liquefactive change, which is a diagnostic feature. Tumors such as myxoid chondrosarcoma are more frequently seen, more aggressive and have a higher propensity for metastases when compared to CMF.

As CMF can show osteoclast-like giant cells at the more cellular zones of the periphery, the possibility of chondroblastoma should also be entertained. As such, chondroblastomas contain uniform cells with clefted or grooved nuclei and eosinophilic cytoplasm with distinct cell borders (chondroblasts). In comparison, CMF shows more stellate nuclear shapes. Also, calcification ("chicken-wire" like) is a conspicuous feature of most chondroblastomas, unlike in CMF [3].Chondroblastoma and CMF do share many similarities, however, including lack of atypia and scant to absent mature hyaline cartilage, but a more separate distinction is not of clinical significance. As a general rule, the term "chondromyxoid fibroma" may be employed if the lesion is in a metaphyseal location and "chondroblastoma" if the lesion is epiphyseal $[5,8]$. 
In the treatment of craniofacial CMF, most authors now recommend thorough curettage of the tumor followed by careful surveillance. However, when curettage is employed, chondromyxoid fibroma shows a recurrence rate approaching $25 \%$ [7]. In many circumstances, especially cases involving the long bones, the preferable treatment is excision, generally due to the functional and cosmetic difficulties following en bloc resection. When the tumor is surgically inaccessible, radiation therapy is indicated $[4,6]$. Local recurrences are more often encountered in younger patients, possibly due to the friable nature of the tumor, but there have been no reports of metastases [8]. There have, however, been reports of the tumor undergoing malignant transformation at a rate of $1-2 \%$, and this seems to have occurred following irradiation $[5,8]$.

\section{References}

1. Lustmann J, Gazit D, Ulmansky M, et al. Chondromyxoid fibroma of the jaws; a clinicopathological study. J Oral Pathol. 1986;15: $343-6$.
2. Ostrowski ML, Spjut HJ, Bridge JA. Chondromyxoid fibroma. In: Fletcher CDM, Unni KK, Mertens F, editors. World health organization classification of tumours: pathology and genetics of tumours of soft tissue and bone. Lyon, France: IARC Press; 2002. p. 243-5.

3. Zilmer DA, Dorfman HD. Chondromyxoid fibroma of bone: thirtysix cases with clinicopathologic correlation. Hum Path. 1989;20: 952-64.

4. Isenberg SF. Endoscopic removal of choindromyxoid fibroma of the ethmoid sinus. Am J Otolaryngol. 1995;16:205-8.

5. Wu CT, Inwards C, O'Laughlin SO, et al. Chondromyxoid fibroma of bone: a clinicopathologic review of 278 cases. Hum Path. 1998; 29:438-46.

6. Rahimi A, Beabout JW, Ivins JC, et al. Chondromyxoid fibroma: a clinicopathologic study of 76 cases. Cancer. 1972;30:726-36.

7. Koay CB, Freedland AP, Athanasou NA. Chondromyxoid fibroma of the nasal bone with extension into the frontal and ethmoid sinuses. J Laryngol Otol. 1995;109:258-61.

8. Armah HB, McGough RL, Goodman MA. Chondromyxoid fibroma of rib with a novel chromosomal translocation: a report of four additional cases at unusual sites. Diagn Pathol. 2007;2: $44-51$. 